\title{
Comparison Between Two Adaptive Controllers Applied to Greenhouse Climate Monitoring
}

\author{
Mohamed Essahafi \\ Preparing a PhD Thesis in Adaptive Control at the Faculty \\ of Sciences and Technology, University of Sultan \\ Moulay Slimane, Morocco
}

\author{
Mustapha Ait Lafkih \\ Professor at the Electrical Engineering Department \\ Faculty of Sciences and Technology, University of Sultan \\ Moulay Slimane, BniMellal Morocco
}

\begin{abstract}
This paper presents a study of a multivariable Adaptive Generalized Predictive Controller and its application to control the thermal behaviour of an agricultural greenhouse, which is composed of a number of different elements (cover, internal air, plants, soil, actuators and sensors). The thermal model was obtained after the study of energy balances reacting the physical behavior of the greenhouse. For this reason, we opted to estimate the dynamic model of the greenhouse with algorithm based on recursive least squares (RLS) method. Simulation results are exposed to show the controller's performances in terms of response time, stability and the rejection of disturbances.
\end{abstract}

Keywords-Generalized predictive control; greenhouse; multivariable control; identification; recursive least square

\section{INTRODUCTION}

The role of the agricultural greenhouse is to produce a crop while avoiding the local climate. It helps to improve the yield of plants [1], and to grow plants that would not survive the natural climate. The most general objective of the producer is to place on the market quantities of agricultural products in relation to the economic demand. For this, he must determine the favorable conditions according to the biological needs of the plant.

The "climate" inside a greenhouse depends on its ventilation. The aeration process is complex, it participates in most of the heat and mass exchanges with the outside, and its control allows to control the physical parameters such as temperature, humidity, or gas concentrations, like $\mathrm{CO}_{2}$ for example. This control is essential to maintain the plants in favorable metabolic conditions (respiration, photosynthesis, transpiration) and in a satisfactory biological state. Adaptive control is necessary to control the greenhouse throughout the functional life of production [2].

In this article we will compare two very famous control strategies, namely, the generalized predictive auto-tuning control (GPC) and the generalized minimum variance (GMV) [3], developed respectively by D. clark 1988, and Astrom and Wittenmark 1973. The GPC control is an extension with an extended horizon of the GMV. This method of control is an approach which has proven its performances in industry. Its algorithm is easier, flexible and robust with respect to other methods. It is applicable to all types of processes, be it variable delay, long or unknown processes, non-minimal phase shift processes, as well as unstable processes (open loop). However, to effectively control the microcilmat in the greenhouse, we chose an adaptive controller that allows online identification of greenhouse parameters and at every moment it calculates the control law that allows to follow in real time the needs of the plant. Although this is based on the recursive least-squares identification (RLS).

To clarify this, we propose in this paper in Section 2, the proposed control approaches GPC and GMV. In Section 3, we present the adaptive control based on online RLS identification method, in Section 4 the simulation results are discussed. Finally in Section 5, a conclusion with future work prospects.

\section{Design of Multivariable Adaptive CONTROLLERS}

\section{A. Greenhouse design}

The control structure [4] chosen for the greenhouse of Fig. 1 shows that the system has two control inputs: heating $(R c)$ and ventilation (Vt), and two outputs to be measured: temperature $(\mathrm{Ti})$ and humidity $(\mathrm{Hi})$, with

$R c$ : heating energy applied to the plant $(K W)$.

$V t$ : ventilation angle outside the greenhouse $(C)$.

To, Ho: air temperature and relative humidity outside the greenhouse $(C, / 100)$.

$S r$ : Solar radiation $(\mathrm{W} / \mathrm{m})$.

$T i, H i$ : air temperature and relative humidity inside the greenhouse $(C, / 100)$.

$S w$ : wind speed outside the greenhouse $(\mathrm{km} / \mathrm{h})$.

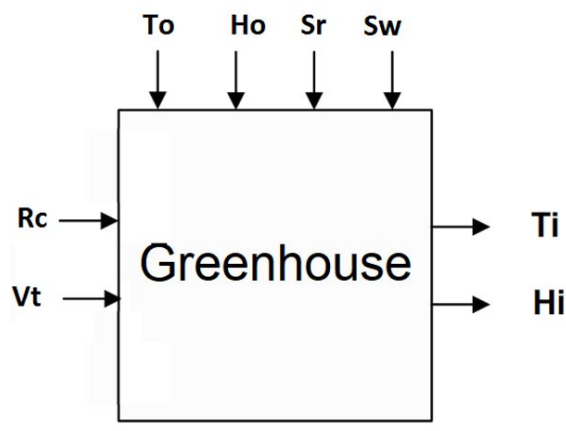

Fig. 1. Schematic diagram of controlled greenhouse.

\section{B. CARIMA Representation}

To describe the discrete behaviour of the greenhouse consider a CARIMA [5] (Controlled Auto-Regressive Integrated 
Media Moving) model

$$
A\left(q^{-1}\right) y(t)=B\left(q^{-1}\right) U(t-1)+\Upsilon(t) \frac{C\left(q^{-1}\right)}{\Delta}
$$

Where $A, B$ and $C$ are polynomials in the back-ward shift operator $q^{-1}$ :

$$
\begin{aligned}
& A\left(q^{-1}\right)=1+A_{1} q^{-1}+\ldots+A_{n_{a}} q^{-n_{a}} \\
& B\left(q^{-1}\right)=B_{0}+B_{1} q^{-1}+\ldots+B_{n_{b}} q^{-n_{b}} \\
& C\left(q^{-1}\right)=1+C_{1} q^{-1}+\ldots+C_{n_{c}} q^{-n_{c}}
\end{aligned}
$$

$\Upsilon$ is an uncorrelated random sequence, $\Delta$ is the difference operator $1-q^{-1}$. For simplicity, $C\left(q^{-1}\right)$ is chosen to be 1 to give the model:

\section{Generalized Adaptive Controller GPC}

To simplify the implementation of the adaptive GPC controller [6], the Diophantine resolution is necessary

$$
A\left(q^{-1}\right) Y(t)=B\left(q^{-1}\right) U(t-1)+\frac{\Upsilon(t)}{\Delta}
$$
$\mathrm{j}$ is

Then the predicted output $Y(t+j)$ for the prediction step

$$
Y(t+j)=E_{j} B \Delta U(t+j-1)+F_{j} y(t)+E_{j} \Upsilon(t+j)
$$

Since $E_{j}\left(q^{-1}\right)$ is a polynomial of degree $j$, the noise components are all at the next discretization steps, so that the optimal predictor, given the measured output data, is explicitly

$$
\hat{Y}(t+j \mid t)=G_{j} \Delta U(t+j-1)+F_{j} Y(t)
$$

Where, $G_{j}\left(q^{-1}\right)=E_{j} B \hat{Y}(t+j \mid t)$

$$
I=E_{j} A \Delta q^{-j} F_{j}\left(q^{-1}\right)
$$

Where, $E_{j} F_{j} A\left(q^{-1}\right)$ Given $A\left(q^{-1}\right)$ denote $\mathrm{A}$ as $\tilde{A}=$ $A \Delta$, such that $\tilde{A}=1+\tilde{A}_{1} q^{-1}+\ldots+\tilde{A}_{n_{a}} q^{-n_{a}+1}$

$$
\begin{gathered}
B=E_{j} B \tilde{A}+B q^{-j} F_{j} \\
E_{j} B=\frac{B\left[1-q^{-j} F_{j}\right]}{\tilde{A}} \\
G_{j}=\frac{B\left[-q^{-j} F_{j}\right]}{\tilde{A}}
\end{gathered}
$$

Consequently, using the recursion of the diophantine equation, so as to obtain the polynomials $E_{j+1}$ and $F_{j+1}$ Considering the values of $E_{j}$ and $F_{j}$ [7]

$$
\begin{gathered}
F_{1}=-\left(\tilde{A}_{1}+\ldots+\tilde{A}_{n_{a}+1} q^{-n_{a}}\right) \\
F_{j}=F_{j, 0}+F_{j, 1} q^{-1}+\ldots+f_{j, n_{a}} q^{-n_{a}}
\end{gathered}
$$

with $i=0,1 \ldots, n_{a}-1$

$$
F_{j+1, i}=F_{j, i+1}-\tilde{a}_{i+1} F_{j, 0}
$$

and for $i=0,1 \ldots, n_{a}-1$

$$
F_{j+1, n_{a}}=-\tilde{a}_{n_{a+1}} F_{j, 0}
$$

Where,

$$
G_{1}=B=B_{0}+\ldots+B_{n b} q^{-n_{b}}
$$

for $n_{G} j=n b+j-1$

$$
G_{j}=G_{j, 0}+G_{j, 1} q^{-1}+\ldots+G_{j, n_{g} j} q^{-n_{g} j}
$$

and for $i=0,1 \ldots, j-1$

$$
G_{j+1, i}=G_{j, i}
$$

Also we can write for $i=j, 1 \ldots, j+n_{b}+1$

$$
F_{j+1, i}=G_{j+i}+B_{i-j} F_{j, 0}
$$

$$
G_{j+i, n b+j}=B_{n_{b}} F_{j, 0}
$$

The term E may be separated by a second Diophantine equation in $\mathrm{Gj}$ and $\mathrm{Hj}$ as follows:

$$
E_{j} B=G_{j}+q^{-j} H_{j}
$$

$$
\begin{gathered}
G_{j}=G_{0}^{j}+G_{1}^{j} q^{-1}+\ldots+G_{j-1}^{j} q^{-j+1} \\
H_{j}=H_{0}^{j}+H_{1}^{j} q^{-1}+\ldots+H_{n_{h}}^{j} q^{-n_{h}}
\end{gathered}
$$

Equation (7) can be rewritten as

$$
\hat{Y}(k+j)=G_{j} \Delta U(k+j-1)+H_{j} \Delta U(k-1)+F_{j} Y(k)
$$

And which considers the following quantities:

$$
\left(\begin{array}{c}
H_{N_{1}} \Delta U(k-1)+F_{N_{1}} Y(k) \\
H_{N_{1}+1} \Delta U(k-1)+F_{N_{1}+1} Y(k) \\
\cdots \\
\cdots \\
H_{N_{2}} \Delta U(k-1)+F_{N_{2}} Y(k)
\end{array}\right)
$$

Adopt a reference sequence $W$ is available. In most cases $W$ will be a constant w equal to the current set-point $W(t)$. The purpose of the predictive control law is to drive the future outputs $Y$ close to future set-point $W$ in some sense [8].

The basic cost function used in GPC has the form [9]

$$
J=(\hat{Y}-W)^{T}(\hat{Y}-W)+\tilde{U}^{T} \Lambda \tilde{U}
$$

with

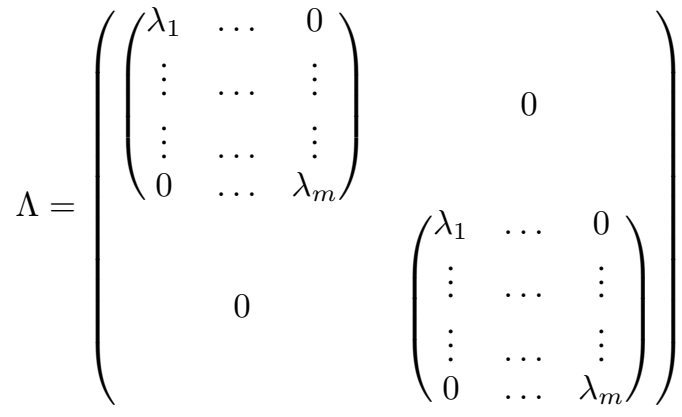


$\tilde{U}=\left[\Delta U(t), \Delta U(t+1)+\ldots+\Delta U\left(t+N_{2}-1\right)\right]^{T}$

and

$$
F=\left[F(t+1), F(t+1), \ldots+, F\left(t+N_{2}\right)\right]^{T}
$$

As cited previously, the first $j$ terms in $G_{j} q^{-1}$ are the parameters of the step-response and therefore $G_{i j}=G_{j}$ for $j<i$.

$\mathrm{G}$ is then a matrix of dimension $\left(m\left(N_{2}-N_{1}+1\right) \times m N_{u}\right.$

$$
\left[\begin{array}{cccccc}
G_{N_{1}-1} & \Lambda & G_{0} & \Lambda & \Lambda & 0 \\
G_{N_{1}} & G_{N_{1}-1} & \Lambda & G_{0} & \Lambda & 0 \\
M & M & 0 & 0 & 0 & M \\
G_{N_{u}-1} & G_{N_{u}-2} & G_{N_{u}-3} & \Lambda & \Lambda & G_{0} \\
M & M & M & M & M & M \\
G_{N_{2}-1} & G_{N_{2}-2} & G_{N_{2}-3} & \Lambda & G_{N_{2}-N_{u}+1} & G_{N_{2}-N_{u}}
\end{array}\right]
$$

Where, $N 1$ and $N 2$ represent minimum and maximum prediction horizons, respectively. Nu represents a control horizon [9].

Given that the first element of $\tilde{u}$ is $\Delta u(t)$ so that the current control $u(t)$ is given by:

$$
U_{\text {opt }}(t)=U(t-1)+\Delta U_{\text {opt }}(t)
$$

with

$$
\Delta U_{o p t}(t)=M 1(W-F c)
$$

The benefit of the GPC algorithm [10] is the expectations made about future control actions. As an alternative of allowing them to be free as for the above improvement, GPC uses the idea that after an interval $N U<N 2$ predictable control steps are supposed to be zero, so we have

$$
\Delta U(t+j-1)=0 \quad, \quad j>N_{U}
$$

\section{Generalized Minimum Variance Controller GMVC}

After seeing the following steps for the design of the GPC controller [11], in the following section we also detail the GMV controller.

Consider the stochastic matrix polynomial model:

$$
A\left(q^{-1}\right) y(t)=B\left(q^{-1}\right) q^{-1} u(t)+D\left(q^{-1}\right) v(t)
$$

is assumed, with

$$
D\left(q^{-1}\right) y(t)=D_{0}+D_{0} q^{-1}+\ldots+D_{m} q^{-m}
$$

A generalized minimum variance controller [12] is obtained by minimizing the criterion.

$$
\begin{aligned}
& I(k+d+1)= \\
& \quad E[y(k+d+1)-w(k)]^{T}[y(k+d+1) \\
& -w(k)]+\left[u(k)-u_{w}(k)\right]^{T} R\left[u(k)-u_{w}(k)\right.
\end{aligned}
$$

With $R=R^{T}$ positive semi-definite $u_{w}(k)$ is the offset steady state value of $u(k)$

$$
u_{w}(k)=B^{-1} A(1) w(k)
$$

Corresponding to (36) the process and signal model is split up into

$$
\begin{aligned}
& q^{d+1} y(t)=A\left(q^{-1}\right)\left[B\left(q^{-1}\right) q u(t)\right. \\
& \left.L\left(q^{-1}\right) v(t)\right]+F\left(q^{-1}\right) q^{d+1} v(t)
\end{aligned}
$$

Where, the new matrix polynomials are defined by:

$$
\begin{gathered}
F\left(q^{-1}\right)=I+F_{1} q^{-1}+\ldots+F_{d} q^{-d} \\
L\left(q^{-1}\right)=L_{0}+L_{1} q^{-1}+\ldots+L_{m-1} q^{m-1}
\end{gathered}
$$

Their parameters are determined by:

$$
D\left(q^{-1}\right)=A\left(q^{-1}\right) F\left(q^{-1}\right)+q^{-(d+1)} L\left(q^{-1}\right)
$$

The term $I(k+d+1)$ is rewritten in the time domain [13], knowing that $[\partial I(k+d+1) / \partial u(k)]=0$ we obtain:

$$
\begin{array}{r}
B_{1}^{T}\left(q^{-1}\right)\left[A\left(q^{-1}\right)\left[B\left(q^{-1}\right) q u(t)+L\left(q^{-1}\right) v(t)\right]\right. \\
-w\left(q^{-1}\right]+R\left[u(k)-u_{w}(k)\right]=0
\end{array}
$$

Where, $\mathrm{v}(\mathrm{t})$ can be reconstructed by:

$$
v(t)=D^{-1}\left(q^{-1}\right)\left[A\left(q^{-1}\right) y(t)-B\left(q^{-1}\right) q^{-1} u(t)\right]
$$

The control vector resulting from the GMV algorithm [14] is written as:

$$
\begin{aligned}
& u(t)=\left[F\left(q^{-1}\right) D\left(q^{-1}\right) B\left(q^{-1}\right) q+\left(B_{1}^{T}\right)^{-1} R\right]^{-1} \\
& \quad\left(I+\left(B_{1}^{T}\right)^{-1} R B^{-1}(1) A(1)\right] w(q) \\
& \left.\quad-A^{-1}\left(q^{-1}\right) L\left(q^{-1}\right) D^{-1}\left(q^{-1}\right) A\left(q^{-1}\right) y(t)\right)
\end{aligned}
$$

If $R=0$ is set, The minimum variance controller result is:

$$
\begin{aligned}
u(t) & =B^{-1}\left(q^{-1}\right) \frac{q^{-1}}{1+q^{-(d+1)}}\left[A\left(q^{-1}\right)[w(t)-y(t)]\right] \\
& \left.+\left[D\left(q^{-1}\right)-L\left(q^{-1}\right)\right] v(t)\right]
\end{aligned}
$$

Where, $v(t)$ must reconstructed from (43). This controller yields for the closed-loop system

$$
y(t)=F\left(q^{-1}\right) v(t)+q^{-(d+1)} w(t)
$$




\section{AdAPtive Control Algorithm}

The general structure of the multivariable adaptive control applied to the agricultural greenhouse is shown in Fig. 2. The online identification of the parameters of the greenhouse is made in such a way as to converge towards the real values using the least squares recursive method [15].

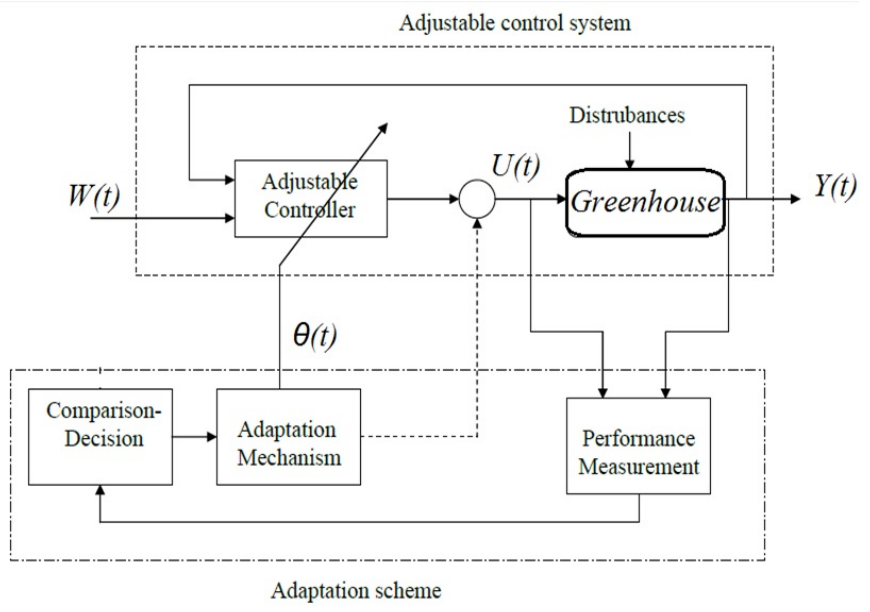

Fig. 2. Adaptive control strategy.

$$
Y(k)=\theta^{T}(k) \varphi(k)
$$

Where, $\theta$ is the vector of the unknown parameters defined as

$$
\theta^{T}(k)=\left[a_{11}(k), \ldots, d_{24}(k)\right]
$$

In (47), $\varphi(k)$ is a regression vector partly consisting of measured input/output variables and is defined as:

$$
\varphi(k)=\left[\begin{array}{llllllll}
T i & H i & R c & V t & \text { To } & H o & S r & S w
\end{array}\right]
$$

The parameter vector is calculated by the following recursive algorithm [11]:

$$
\begin{gathered}
\theta(k)=\operatorname{Argmin} \sum_{i=1}^{k} \lambda^{k}\left(\left(y(k)-\varphi^{T} \widehat{\theta}(k)\right)^{2}\right. \\
\widehat{\theta}(k)=\widehat{\theta}(k-1)+K(k)\left[\left(y(k)-\varphi^{T} \widehat{\theta}(k-1)\right)\right] \\
K(k)=\frac{P(k-1) \varphi(k)}{\lambda+\varphi^{T}(k) P(k-1) \varphi(k)} \\
K(k)=\frac{1}{\lambda}\left(P(k-1)-K(k) \varphi^{T}(k)\right)
\end{gathered}
$$

\section{Simulations AND RESUlts}

After identifying the agricultural greenhouse by a mathematical model in space state, the validation phase of the model by the simulations made on the greenhouse is necessary. The purpose of this paragraph is to test in simulation mode the control law multivariable predictive GPC on the identified discrete model of the greenhouse and compare it with the multivariable GMV control.

Then, the discreet model of the greenhouse is written by:

$$
\begin{gathered}
A 1=\left[\begin{array}{ll}
-0.1 & 0.2 \\
0.33 & 0.4
\end{array}\right] \quad ; \quad A 2=\left[\begin{array}{cc}
-0.5 & 0.66 \\
-0.77 & 0.8
\end{array}\right] \\
B 0=\left[\begin{array}{ll}
-0.1 & 0.2 \\
-0.3 & 0.4
\end{array}\right] \quad ; \quad B 1=\left[\begin{array}{cc}
0.5 & -0.6 \\
-0.7 & 0.8
\end{array}\right] ; \\
B 2=\left[\begin{array}{cc}
0.9 & -0.1 \\
0.11 & -0.12
\end{array}\right]
\end{gathered}
$$

The subsequent experiments represent variations of humidity and temperature set points in the greenhouse process. In general, each trial track can be separated into three sequential phases. Through the first, start-up phase the system plant is achieving steady state around the operation points. The second one is a suitably chosen identifying phase using acquired data, which gives us an initial estimation of CARIMA model parameters. The third phase finally displays the results of the adjusted multivariable adaptive GPC controller. Several of realtime tests had been simulated in order to choice parameters that would offer the desired controller performance. As a final point, the succeeding values of design parameters were set.

- For adaptive GPC control sampling times $T=1.5 \mathrm{~s}$, horizons $N 1=1, N 2=10, N u=4$, dead time $d=3$ and weights $[\lambda 1, \lambda 2]=[0.97,0.95]$

- For the cases using GMV as the control algoritlim, $R=\left[\begin{array}{cc}10 & 1 \\ 5 & 10\end{array}\right]$ is used.

In the following figures we present the simulations made for the two adaptive controllers. For the GMV controller the influence of the multivariable coupling of the parameters of the greenhouse is clearly felt on the first control at the time of the change of the setpoint applied to the second output (Fig. 3 and 4). The first control reacts so that the air temperature is disturbed by the change in behavior that affects the airflow.

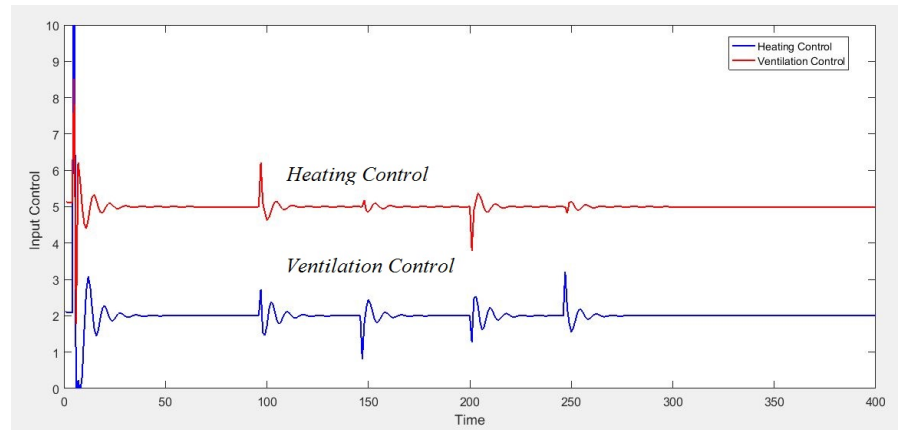

Fig. 3. The input control of the greenhouse with the AGMV controller. 


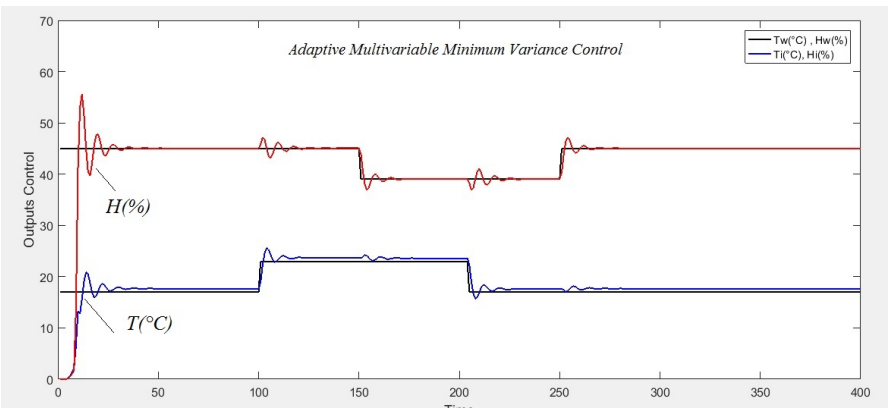

Fig. 4. Responses of greenhouse air temperatures and relative humidity using the AGMV controller.

With the observation of Fig. 3 and 4 for the GPC controller, we note despite the presence of the coupling between the variables of the thermal process, the set point change in real time on both the first output (top) and the second output (bottom) has less impact on both outputs. The inputs controls applied to the system are given in Fig. 5.

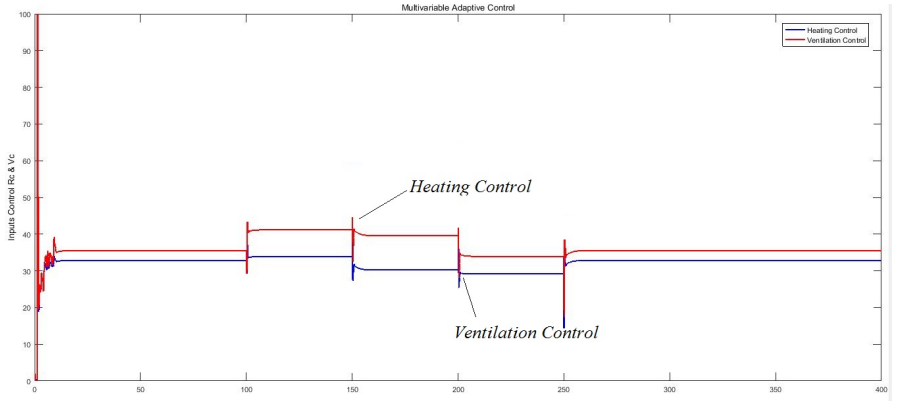

Fig. 5. The input control of the greenhouse with the AGPC controller.

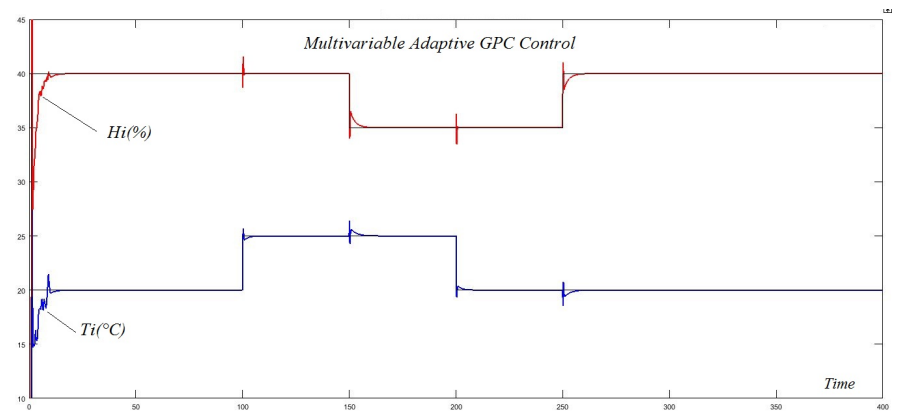

Fig. 6. Responses of greenhouse air temperatures and relative humidity using the AGPC controller.

The evolution over time of the dynamic parameters of the greenhouse is illustrated in Fig. 6. These parameters are initialized to values less than unity, but they are then recalculated taking into account the thermal behavior of the greenhouse. When the disturbed load is applied, the parameters are again re-estimated.

The aim purpose of this study was to compare the performance of the two adaptive controllers AGPC and AGMV for disturbance rejection as well as set-point tracking. The various simulation tests show that the GPC adaptive controller is more powerful than the GMV adaptive controller. In fact, the output response of the greenhouse follows the fixed setpoint for both controllers as shown in the figures Fig. 4 and 7. The only difference is the way in which the output arrives at the setpoint.
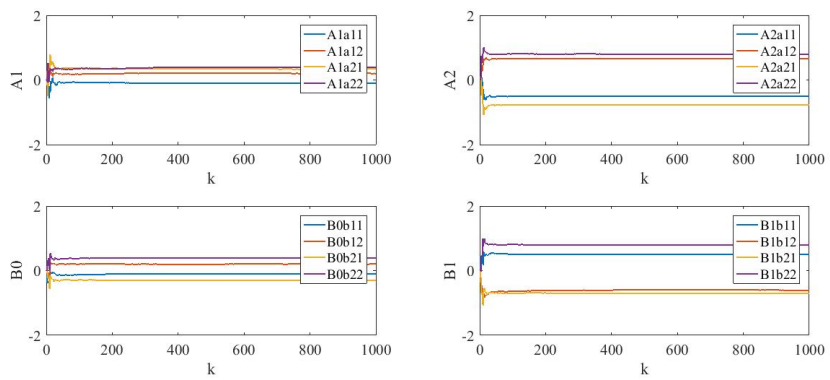

Fig. 7. The online estimation of the greenhouse's parameters A1, A2, B0, B1.

Moreover, for the GPC controller the output is characterized by a better stability and a lack of the oscillations of overtaking by comparing to the GMV controller, while the control effort is better for the GMV controller.

\section{CONCLUSiON}

A Summary Comparison of GPC Adaptive Controllers and GMV is rigorously implemented for controlling the temperature and humidity of the air inside the environment of a greenhouse, based on least-square estimation technique. The control objective is to enforce the air temperature set point within the greenhouse to track its desired value. It appears, using real results and simulation that the GPC adaptive controller retains so much stability and robustness in comparison with the GMV adaptive controller.

As future work, the global microclimate of the greenhouse taking into account the external and internal disturbance will be developed, in order to meet the basic needs of greenhouse cultivation.

\section{REFERENCES}

[1] N. Dinesh Kumar, S. Pramod, C. H. Sraboni, Intelligent irrigation system, International Journal of Agricultural Science, vol. 3, pp. 23-30, August. 2013.

[2] Li. XH, X Cheng, K Yan, P. Gong, An monitoring system for vegetable greenhouse based on a wireless sensor network, PubMed journal on Sensors (Basel), vol. 10, pp. 8963-8980, October 2010.

[3] Astrom, K. J., and B. Wittenmark, ,Adaptive Control Addison-Wesley Publishing CompanySecond Edition,, 1995.

[4] Lauri, J. V. Salcedo, S. Garcia-Nieto and M. Martinez,Model predictive control relevant identification: multiple input multiple output against multiple input single output in IET Control Theory and Applications, vol. 4, no. 9, pp. 1756-1766, September 2010. doi: 10.1049/iet-cta.2009.0482

[5] K. P. Lam, On Using the Filtered CARMA and CARIMA Models for State-Space Self-Tuning Control 1987 American Control Conference, Minneapolis, MN, USA, 1987, pp. 1286-1290.

[6] Clarke, D. W., C. Mohtadi, and P.S. Tuffs, Generalized Predictive Control Part 1. The Basic Algorithm; Part II. Extensions and Interpretations, Automatica, Vol. 23, No. 2, pp. 137-160, 1987.

[7] M. J. Grimble, P. Majecki and L. Giovanini, Polynomial approach to nonlinear predictive GMV control 2007 European Control Conference (ECC), Kos, 2007, pp. 4546-4553.

[8] D. W. Clarke, P. J. Gawthrop (1975), Selftuning control, Proc.IEE, vol. 26, no. 6, pp. .Part D: Control Theory and Applications 122(9), 929-934 [Seminal paper on GMV-based self-tuning control]

[9] D. W. Clarke, P. J. Gawthrop, SelfTuning controller", Proc.IEE, vol. 122, no. 9, pp. Control Theory and Applications 126(6), 633-640, 1979.[A review of GMV-based self-tuning control.]

[10] Z. Zidane, M. Ait Lafkih and M. Ramzi, Application of Multivariable Linear Quadratic Gaussian Control and Generalized Predictive Control in a Hydropower Plant, International Journal on Sciences and Techniques of Automatic control and computer engineering IJ-STA, vol. 7 no. 1, April (2013), pp. 1890-1906.

[11] S. C Chai, G. P. Liu and D. Rees, Design and implementation of networked predictive control systems, 16 IFAC World Congress, Prague, 2005 
[12] G. A. Montague, A. J. Morris and M. T. Tham,Performance evaluation of three multivariable self-tuning controller design techniques 198625 th IEEE Conference on Decision and Control, Athens, Greece, 1986, pp. 1564-1569. doi: 10.1109/CDC.1986.267148

[13] G. A. Montague, M. T. Tham and A. J. Morris, A Comparison of Multivariable Long Range Predictive Control with GMV Control in a Highly Nonlinear Enviroment 1986 American Control Conference,
Seattle, WA, USA, 1986, pp. 721-727.

[14] M. J. Grimble and P. Majecki, Nonlinear Predictive GMV control 2008 American Control Conference, Seattle, WA, 2008, pp. 1190-1195. doi: 10.1109/ACC.2008.4586654

[15] J. E. Bobrow and W. Murray, An algorithm for RLS identification parameters that vary quickly with time in IEEE Transactions on Automatic Control, vol. 38, no. 2, pp. 351-354, Feb 1993. doi: 10.1109/9.250491 